\title{
ROBINSON CRUSOE Y SU MOZO MORISCO XURY: DANIEL DEFOE Y EL MUNDO HISPÁNICO
}

\author{
Leonard P. Harvey*
}

Cuando en 1719 Daniel Defoe publicó su gran novela Robinson Crusoe, ésta tuvo enseguida un gran éxito con el público ${ }^{1}$. El libro gana pronto una fama mundial. Rousseau (en Émile) consideraba que debería ser el primer libro leído por un joven, Marx lo cita en Das Kapital, etc. Pero si todo el mundo sabe algo de lo que le sucede a este marinero en su isla "cerca de la embocadura del gran río Orinoco" (y sobre todo nos acordamos de las relaciones entre Crusoe y el hombre que rescató de la muerte, y que vino a ser su fiel Viernes), nadie parece acordarse del incidente cerca del comienzo de la novela que es el motivo de estas líneas. No pretendo que la muestra de mis amigos y colegas a quienes he dirigido mis preguntas sobre este particular sea estadísticamente satisfactoria, pero sin excepciones parece que se ha producido entre ellos un tipo de amnesia colectiva. Me refiero al incidente de Crusoe y el morisco llamado Xury, del cual nadie parece acordarse. Y me pregunto si el hueco que se ha producido en nuestra memoria no nos dice algo acerca de las actitudes del mundo occidental no sólo hacia el problema de los moriscos, sino hacia las minorías musulmanas en general.

Si nadie recuerda bien el incidente, me perdonarán si esbozo un breve resumen de lo que nos dice el libro:

El joven Robinson Crusoe no quiso plegarse a los deseos de sus padres y buscar un empleo seguro en el condado de Yorkshire, donde había nacido en 1632: se lanza a la peligrosa aventura del comercio con el África Occidental (Guinea). Del éxito de su primer viaje sacó un modesto capital que no vaciló en invertir en un segundo (como observa Marx², las experiencias de

* Oxford Centre for Islamic Studies.

1. La portada de la primera edición no menciona el nombre del autor, pero no existe ninguna duda sobre la autoria de Defoe: The Life and Strange Surprizing Adventures of Robinson Crusoe of York, Mariner (etc.) Written by Himself, London, printed for W. Taylor at the Ship in Paternoster-Row. MDCCXIX.

2. Cito según la traducción inglesa de S. Moore y E. Aveling, Capital, Chicago, 1921, p. 88. 
Crusoe son un tema predilecto de los estudiosos de la economía política, y no es difícil ver por qué). Y el desastre no tardó en producirse. "En el gris del amanecer" ("In the grey of the morning") "mientras que nuestro barco se dirigía hacia las Islas Canarias, o, mejor dicho, entre aquellas islas y la costa africana, fue sorprendido por un corsario saletino turco, quien se lanzó a perseguirnos con todas las velas que podía izar". El barco inglés es capturado. Los tripulantes son llevados a Marruecos, y la mayoría de ellos destinados al servicio de la corte del rey, pero el capitán pirata guarda al joven Crusoe como esclavo doméstico. En Salé, "puerto de mar perteneciente a los moros", además de trabajar en las faenas domésticas ("drudgery") demuestra gran destreza cuando sale con su amo en la lancha en sus expediciones de pesca. Y esta circunstancia le proporciona la ocasión de evadirse. En el día en cuestión iban con él en la lancha, para vigilarle seguramente, dos musulmanes. Uno era un adulto, miembro de la misma familia del pirata moro, Muley Ismael; el otro, un jovencito, se describe como un "Morisco" 3 .

Éste estaba remando, mientras que Ismael estaba sentado en la proa del barco. Crusoe se le acercó, y de pronto lo agarró, lanzándolo fuera del barco. Bajo las amenazas de una escopeta a la que Crusoe había echado mano, el moro no tuvo más remedio que alejarse hacia la costa, nadando. Crusoe ahora necesita reclutar al morisco, Xury, como tripulante. Nos confiesa que hubiera preferido echar al morisco al agua, guardando con él al moro (adulto) pero ¿cómo estar seguro de él? ("there was no venturing to trust Ismael"). De modo que se dirige al mozo, dándole la alternativa: "o prometes serme fiel, en cuyo caso te haré un gran hombre, o te echo al mar también". EI mo-

3. La primera edición, así como las subsiguientes, lo llama 'Maresco', y es de notar que la traducción al francés, aparecida aquel mismo año en Amsterdam, muestra 'Maresco' también. Ahora bien, se plantea la cuestión de si se trata de una forma que sale de las intenciones del mismo autor o si 'Maresco' al principio no era sencillamente una mala lectura -en la imprenta o en otro sitio-. En la escritura cursiva de muchas personas la 'a' y la 'o' serían confusibilia (y 'moresco' sería sencillamente una forma italianizante). Lo que es quizá inquietante es que un tal Peter Maresco (sic) tuvo un papel crucialmente importante en la vida de Defoe. Entre los nombres de los acreedores que le ocasionaron la bancarrota a Defoe en 1699, figura en primer lugar (con una deuda de $£ 400$ ) este hombre. Dado que al ficticio Crusoe le es dado un apellido "real", ¿tendríamos aquí otro ejemplo del mismo fenómeno? De todos modos está fuera de duda que en el Salé del siglo XVII había dos grupos, el de los nativos originales, a los que convendría perfectamente el epíteto empleado por Defoe, "moro": el amo de Crusoe y sus parientes como Ismael eran moros. El otro grupo era el de los moriscos, llegados de España como refugiados a raíz de la expulsión de 1609-1612, de Hornachos muchos de ellos. Cómo se convirtieron pronto en una entidad de peso en la ciudad, hasta el punto de formar una república independiente, en conflicto a veces con las autoridades marroquíes ( $\sin$ hablar de sus depredaciones contra la marina mercante de varios países occidentales), constituye un capítulo interesante en la historia de Marruecos. Si en Salé Crusoe encuentra a moros, y a uno que es en cierto modo distinto de ellos y que se llama 'maresco', no cabe duda de que se trata de un morisco, sea como fuere la forma de la palabra utilizada de hecho por Defoe. Parece plenamente justificable la decisión de muchos traductores del texto al español de decir "morisco". 
risco acepta, sonríe, "y habló con tanta inocencia que me era imposible no fiarme de él". De esta manera Crusoe adquiere el tripulante que necesita, y en lo que sigue, Xury cumple con lo prometido. La verdad es que hace más, y en varias ocasiones durante el viaje del pequeño barco hacia el sur, pone su vida en peligro al servicio de este amo cristiano. Es él, por ejemplo, quien mata a un león enorme dándole el coup de grace por la oreja con una pequeña escopeta. El morisco le muestra a Crusoe "tanto afecto que me hizo quererle siempre" ("made me love him ever after"). "Siempre" aquí significa de hecho, según nos damos cuenta más tarde, "varios meses". Porque les salva un barco portugués, rumbo al Brasil. Es típico que sea Xury quien divisa el barco primero. "Mi amo, un barco con una vela". El capitán del barco los lleva con él. Y una vez llegados al Nuevo Mundo, para ayudar al marinero inglés, y proveerle de un pequeño capital para establecerse en la colonia portuguesa, le compra a Crusoe la lancha pesquera con sus enseres, y no sólo el barco. "Me ofreció además sesenta piezas de a ocho en cambio de mi mozo (boy) Xury. No deseaba aceptarlas ("which I was loath to take"), y esto no porque no quisiera cederlo al capitán, sino porque no quería vender la libertad de alguien que me había servido con tanta fidelidad mientras yo conseguía la mía. Pero cuando le expliqué mis razones al capitán, las aceptó como bien fundadas, sugiriéndome esta avenencia: que se comprometería libertar al mozo, con tal que se convirtiera al cristianismo. Con esta promesa, y con el acuerdo de Xury, que estaba dispuesto a pasar a su servicio, lo cedí al capitán". (Es de notar que no se menciona para nada aquí aquellas palabras pronunciadas antes por Crusoe: "Te haré un gran hombre". Varios años como esclavo personal del capitán, por muy agradable que fuera, no representaban el cumplimiento de tal promesa.)

Y desde aquel momento sólo se menciona a Xury una vez. Cuando Crusoe empieza a tener cierto éxito en su hacienda brasileña, se da cuenta que el factor que le limita es la falta de mano de obra. "Y ahora me puse a pensar más que nunca que había hecho mal separándome de mi mozo Xury". ¿Qué se hizo del mozo? No lo sabemos. ¿Acepta la libertad a cambio de ser convertido? Hacia el final de su novela Defoe se empeña en atar muchos cabos sueltos de este tipo. ¿Qué se hizo, por ejemplo, de su paraguas, construido con tanta ingeniosidad? ¿Del papagayo que le hablaba cuando no tenía a nadie con quien hablar? Defoe nos lo dice (se los llevó a Inglaterra). Pero sobre el morisco, ni una palabra. Aun cuando por fin puede dejar su isla, y se le ocurre pensar en el lapso de tiempo desde su evasión de Salé: "el 19 de diciembre de 1686, después de haber estado en la isla 28 años, dos meses y 19 días, liberado así de ésta mi segunda cautividad, y el mismo día del mes que el de mi fuga en la lancha de entre los moros de Salé". Es imposible imaginar que se haya acordado así de su evasión sin haber pensado en el tripulante que le había servido fielmente. Y más tarde, cuando descubre que el viejo capitán portugués vivía en Lisboa, hace un esfuerzo y va a visitarle: naturalmente se ponen a hablar de los viejos tiempos. Pregunta por la 
hacienda, por sus socios y amigos. Ni una palabra acerca del mozo (cuya suerte había dependido de la buena voluntad del capitán).

El tratamiento tan cruel del mozo morisco, su rechazo y abandono, nos lleva a plantear la cuestión de si este texto hay que interpretarlo como irónico o no. No cabe duda: Defoe era perfectamente capaz de emplear la ironía, y hasta podríamos decir que era uno de sus recursos preferidos. En el caso del panfleto The shortest way with Dissenters ("El modo más rápido de terminar con los No-conformistas") esta característica suya le llevó a la pública vergüenza de verse expuesto en los cepos. No se trata aquí de entrar en una detallada descripción de lo que podría describirse como uno de los más gloriosos días de la vida de nuestro autor, porque la vergüenza se convirtió casi en un triunfo, pero sí conviene por lo menos mencionar lo ocurrido para ilustrar la importancia de la corrosiva ironía en su estilo. Había escrito el panfleto en cuestión para satirizar la actitud de los miembros de la tendencia anglicana denominada "High Church" (Iglesia Alta, o sea, el ala menos "protestante" de la Iglesia Anglicana) frente a los así llamados Dissenters, protestantes cien por cien, gente que se negaba a aceptar las disciplinas y obligaciones de la iglesia nacional. Ahora bien, la base de la sátira de Defoe estribaba en hablar como si fuera un adepto de esta tendencia "High Church", y en abogar en pro de la expulsión de tales protestantes peligrosos, no sólo de la iglesia, sino del país entero. Algunos enemigos de los Dissenters no veían la ironía que había detrás de las palabras de Defoe, y aplaudían. Mientras que muchos de los Dissenters tampoco entendían la intención irónica del panfleto, y se quejaban. El gobierno sí que comprendía la ironía perfectamente, pero la interpretaba como una amenaza contra el orden público, y el pobre Defoe terminó en la cárcel. Se le acusaba de "seditious libel" ("difamación sediciosa"), y ya que antes del proceso sus amigos le habían aconsejado no intentar defenderse, Defoe tuvo que pagar una multa importante y se vio condenado a varias horas en los cepos (pillory). Cómo salió airoso del apuro no hace al caso ahora, pero sí es importante tener en cuenta esta demostración de cuán fácil es interpretar mal los sarcasmos de este autor.

Si sus contemporáneos eran capaces de interpretar mal The Shortest Way with Dissenters, debemos proceder con cautela con el pasaje que nos interesa aquí, porque es posible que, después de tres siglos, interpretemos mal el tono de Defoe. ¿Hay quizá detrás de lo que nos cuenta acerca del morisco Xury una crítica del tratamiento de este grupo humano? Para ayudarnos a decidir entre las dos posibilidades tenemos por un lado el hecho de que haya militado durante su vida entera en pro de la tolerancia, así como su conocida preferencia por la ironía. Pero por otro lado hay que tener en cuenta sus opiniones acerca del Islam. Su tolerancia abarca las varias manifestaciones de la fe cristiana, y hasta a veces muestra cierta comprensión frente al paganismo, pero del Islam es un enemigo implacable. En An appeal to Honour and Justice (escrito en 1715, pero utilizando una obra suya anterior), en una referencia al 
sitio de Viena de 1683, habla de la historia de los Turcos como de una serie de "tratos crueles y pérfidos"4. Habían "desarraigado el nombre de la religión cristiana de más de setenta reinos", de modo que en su opinión los ingleses tenían la obligación de postergar su natural enemistad hacia el Santo Imperio Romano en pro de la necesaria defensa de Europa. El tratamiento tan duro recibido por Xury se explica acaso a la luz de estas actitudes.

Todo depende, claro está, de nuestra interpretación de las intenciones subyacentes del autor $\mathrm{y}$, para llegar a conocerlas, necesitamos examinar algunos ejemplos de su comportamiento en el resto de su vida. Pero aquí estamos pisando un terreno resbaladizo. Tenemos que ver con un hombre que se nos escapa cada vez que intentamos asirle. Defoe era un moralista dedicado a la educación del gran público, y era también un estafador acusado de fraude. Lo que es más, durante buena parte de su vida ganó dinero facilitando informes al servicio secreto de su Majestad: hablando llanamente, era un espía del gobierno. Este mismo aspecto doble, esta ambigüedad, la encontramos en otros sectores de su ingente obra. Tomemos tan sólo un ejemplo. En 1722 como moralista y educador escribió Religious Courtship: being Historical Discourses on the necessity of marrying religious husbands and wives only, as also of husbands and wives being of the same opinion in religion as one another. Al cabo de un mes salió de la imprenta otra obra suya, esta vez una novela titulada The Fortunes and Misfortunes of the famous Moll Flanders, quizá su obra que mejor se vende hoy en día. Suele encontrarse en los estantes de las librerías al lado de obras de pornografía y de tratados de sexología. Yo no intento clasificar esta obra, sólo doy fe de la opinión de muchos libreros de nuestros días.

\section{LA BIOGRAFÍA DE DEFOE}

¿Cómo vino a ser lo que fue? Nacido en Londres en el año 1660, su juventud coincide con la restauración en el trono de los Estuardos después de los movidos años de la República (Commonwealth) de Cromwell y, más tarde, en 1688 ( $\mathrm{y}$ al otro lado de la barrera) vive la así llamada "Gloriosa Revolución", mediante la cual se reemplazó al "catolizante" James II por la menos peligrosa Mary, casada con el holandés Guillermo de Orange. Y su vida se termina bajo los Georges de la casa de Hannover. Época tumultuosa en la que todavía había revoluciones y batallas campales en el suelo de Inglaterra.

El factor más importante en la formación intelectual de Defoe fue su condición de "dissenter", es decir, miembro de una de las muchas sectas

4. BACKSCHEIDER, pp. 44-45. 
protestantes que se negaban a aceptar la disciplina de la Iglesia Anglicana. La consecuencia de este rechazo de las doctrinas del régimen fue la discriminación. Si bien es verdad que si comparamos la suerte de estos ultra-protestantes con lo sucedido en otros países europeos en aquella época, vivían tranquilamente. No temían la muerte en la hoguera ni la tortura, pero se les negaba el acceso a muchas capas de la vida pública. Los que no querían comulgar de vez en cuando en una iglesia anglicana ("occasional conformity") no podían matricularse, por ejemplo, en las universidades de Oxford y de Cambridge (y no había otras entonces). $Y$ sin los correspondientes grados no podían acceder a muchos puestos. Por esta razón la inteligencia ultra-protestante tendía a dirigirse hacia actividades más independientes, y sobre todo hacia el comercio. Pero si Defoe nunca acudió a las aulas de Oxford, no por esto dejó de recibir una formación muy sólida, en una de las "dissenting academies", o sea, en uno de los colegios a modo de seminarios que servían para formar a los pastores protestantes y, al mismo tiempo, daban una excelente educación general a los hijos de la burguesía ultra-protestante. (En el caso de Defoe, la "academy" en cuestión era la de Newington Green, bajo la dirección del famoso Charles Morton, hombre que más tarde pasó al Nuevo Mundo, donde tuvo puestos de cierta relevancia.)

Otro factor de gran importancia en la vida de este hombre tan complejo fue el papel que desempeñó como espía al servicio del gobierno. Otra contradicción. Para comprender cómo llegó a ser un agente conviene tener en cuenta sus constantes apuros económicos. Defoe, siempre emprendedor, se lanzó ya en su juventud a mil proyectos. Lo que le hacía falta era el capital, y, fuerza es decirlo, la prudencia. En el año de 1692 perdió mucho dinero intentando sin éxito perfeccionar una campana de buzo ideada por Sir Edmund Halley para extraer de un buque naufragado en la costa una cantidad de plata. El proyecto sólo generó deudas, y acusaciones de fraude y de malversación de fondos. $\mathrm{Y}$ en otros proyectos posteriores Defoe llegó a perder más dinero, dinero que era suyo, dinero de su pobre mujer, dinero de otros... En 1692 estaba ya en la cárcel del Fleet. Se le licencia temporalmente, pero no llega a salvar sus empresas. Su situación se hizo imposible: a sus deudas comerciales (explicables en gran parte por las demoras en el pago de lo que se le debía a él) se sumaban las multas que se le iban infligiendo por incumplimiento de las decisiones de los tribunales.

Lo que le salvó a Defoe de la cárcel fue algo que podría figurar sin modificación alguna en una novela de espías y agentes secretos. Harley, ministro principal del gobierno (todavía no podemos hablar de "primer ministro", el primer "primer ministro" en el sentido moderno fue Walpole, unos pocos años después), empezó a interesarse por este intelectual que se encontraba en la cárcel. Pero su ayuda no era desinteresada. Comprendió que el "dissenter" podría serle útil. Exigió un precio. El ministro autorizó el desembolso de los fondos oficiales de cierta cantidad de dinero a un asociado de Defoe, y 
nuestro autor se encontró en libertad". De este modo el "dissenter" tan independiente vino a verse atrapado en el engranaje del servicio real.

Dentro de su propia sociedad Defoe ocupaba, pues, una situación privilegiada de "insider", de persona arrimada a los centros del poder, y al mismo tiempo de "outsider", de ser marginado por múltiples razones, confesionales, económicas, psicológicas.

\section{DEFOE Y EL MUNDO HISPÁNICO}

Pasemos ahora a la actitud de Defoe frente al mundo hispánico. Y podemos empezar en el epicentro del poder en la Inglaterra de entonces, en un proyecto presentado al rey Guillermo de Orange. "I had the honour to lay a proposal before his late majesty King William in the beginning of the war for settling the English on the coasts of South America" (Defoe, Review, 8:165, citado por Seidel, p. 41): "Tuve el honor de presentarle a nuestro lamentado rey Guillermo al principio de la guerra [entiéndase la de la Sucesión Española] un proyecto para crear colonias de ingleses en las costas de América del Sur", anuncia con cierto orgullo.

El interés expresado de este modo en extender al hemisferio sur la actividad colonializadora inglesa ya desarrollada en América del Norte tiene muchos paralelos en otros lugares de su obra. Pero es fácil interpretar mal estos aspectos de su programa político. Los protestantes de la tendencia de los Dissenters eran con frecuencia muy anti-papistas y, por lo tanto, muy enemigos de los españoles. Esta característica perdura aún hoy en día en la iconografía de los barrios populares protestantes de Belfast (Ulster), donde precisamente el buen rey don Guillermo (montado en un caballo) aparece como símbolo de la resistencia al Papa (el icono habrá salido en infinitos reportajes televisivos). Pero Defoe no era así. Lo que él temía era la intromisión de los franceses en tierras americanas y en el mundo hispánico. El vacío creado por la debilidad militar de España lo veía como un problema geopolítico. Pero no buscaba causar la destrucción del imperio español. Al contrario. "Where will we be when a French garrison is planted at Cadiz?", pregunta. O: "What will the Virginia colony be worth when the French have free commerce from Quebec to Mexico behind ye?" (¿Qué será de nosotros con una guarnición francesa establecida en Cádiz? - ¿Qué valdrá la colonia de Virginia cuando el comercio francés circule libremente desde Quebec hasta

5. Curiosamente, existe, según la biografía de Backscheider, una nota marginal en el registro oficial de cuentas precisando que el pago se efectuó por orden del ministro, sin otra justificación que el servicio secreto de su Magestad: "My lord Treasurer directed this sum be paid him for Her Majesty's Secret Service, and he would give no other acq(uittan)ce". (BACKSCHEIDER, p. 123.) 
México?) ${ }^{6}$. Lo que Defoe quería era que se llegara a crear una avenencia ventajosa a ambas partes entre España y Inglaterra: y contra Francia. A los españoles (y a los portugueses también) los veía como los aliados más indicados de los ingleses.

Estas ideas se transparentan en el argumento de Robinson Crusoe. Crusoe en su isla está rodeado por peligrosos enemigos caníbales. Llevan a sus cautivos a la isla para comérselos allí. Crusoe le salva la vida a uno de estos prisioneros, y la primera palabra que pronuncia el hombre cuando Crusoe le corta sus ataduras es "Christianus". El hombre resulta ser un español. Crusoe le da armas, y ambos se ponen a combatir con éxito a los enemigos del español, es decir a los enemigos de España. De las autoridades españolas existentes en la región, repite con aprobación la opinión del español: "They were all of them very civil honest men" (eran todos muy corteses y honrados). La imagen de los portugueses es, si cabe, aún más favorable. Gracias a la honradez y amabilidad del capitán portugués puede adquirir su finca, gracias a la honradez de las autoridades portuguesas que siguen administrándola en su nombre, dispone, después de los muchos años de su ausencia de la isla, de un bonito capital que le permite retirarse. ¿Cómo dramatizar de una manera más eficaz los posibles provechos de la cooperación y de un acuerdo anglo-ibérico? Y es de notar que Defoe, dissenter, nos cuenta en un tono aprobatorio que una proporción de los beneficios de las empresas se había dedicado a la conversión de los paganos de la región. Queda por decir que los misioneros habían sido católicos. Crusoe, y detrás de él Defoe, no es ningún antipapista obcecado.

Es a la luz de la presentación positiva de los "ibéricos" en esta novela como hay que leer e interpretar lo que pasa con Xury. En mi opinión el episodio no se explica como un ataque satírico e irónico contra el tratamiento de los moriscos. Los polemistas ingleses eran perfectamente capaces de aprovechar este tema de los moriscos como un capítulo más de la leyenda negra (como M. de Epalza ha demostrado muy bien en sus estudios sobre Morgan y Geddes ${ }^{7}$ ). Pero el argumento de Defoe en Robinson Crusoe es de otra índole. Lo que busca es la mejora de las relaciones entre Inglaterra y el mundo hispánico. Explícitamente no se menciona el problema de los moriscos, que había sido resuelto de una manera tan determinante 110 años antes de la publicación del libro, pero implícitamente se acepta lo hecho. Y de ahí el tratamiento de Xury. Si Defoe buscaba mejorar las relaciones entre los dos países, no era la hora de sacar este cadáver del armario.

6. SEIDEL, p. 41.

7. Míkel de EpalzA, «Relaciones del cónsul británico Morgan con descendientes de moriscos en el Mágreb (siglo XVIII)», Estudios de Filología Inglesa: Homenaje al Dr. Pedro Yesús Marcos Pérez, Alicante, Universidad de Alicante, 1990, pp. 615-620. 
Si mi interpretación de este pasaje es correcta (y quisiera hacer hincapié en esto: se trata de una apreciación personal), Defoe comparte con la mayor parte del mundo occidental lo que podría llamarse nuestra amnesia colectiva sobre este fenómeno del tratamiento de la minoría islámica que eran los moriscos. Para los españoles, así como para los demás europeos occidentales, los moriscos habían, simplemente, desaparecido de la escena, y esto era todo. En la historia ya no existían y por lo tanto habian perdido sus derechos. Xury al final es vendido, y puede desaparecer de la narración. Crusoe pretende aclarar que la venta no la hizo de buena gana ("I was loath etc."). No sería difícil encontrar paralelos a esto en nuestros tiempos modernos ${ }^{8}$.

\section{BIBLIOGRAFÍA SELECTA}

Backscheider, Paula, Daniel Defoe: His Life, Baltimore and London, The Johns Hopkins U.P., 1989.

Defoe, Daniel, Robinson Crusoe: an Authoritative Text, Contexts, Criticism, edited by Michael Shinagel, New Yok, London, W.W. Norton, 1994.

Defoe, Daniel, Defoe's Review, ed. A.W. Secord, 9 vols., New York, Columbia U.P., 1938.

Furbank, P.N. and Owens, W.R., Defoe De-Attributions, London and Rio Grande, The Hambledon Press, 1994.

Healey, G.H., The Letters of Daniel Defoe, Oxford U.P., 1955.

Moore, John Robert, A Checklist of the Writings of Daniel Defoe, Bloomington, Indiana U.P., 1960.

SeIDel, Michael, Robinson Crusoe, Island Myths and the Novel, Boston, Twayne, 1991.

\section{RESUMEN}

Daniel Defoe publicó Robinson Crusoe en 1719. Crusoe, en las primeras aventuras que corre antes de llegar a su isla, tiene en una ocasión que escapar de la cautividad en Salé (Marruecos), donde es auxiliado por un "moro". Consigue fugarse en un barco de su amo con la ayuda de un mozo llamado Xury, que puede ser identificado con garantía como un morisco ("maresco" en el texto). Pero Crusoe, que le había prometido hacerlo "un gran hombre", termina por venderlo por sesenta piezas de a ocho a un capitán portugués. El artículo sostiene que el episodio, más que por un criticismo irónico de la conducta de Crusoe, se explica por la defensa de Defoe de unas mejores relaciones con el mundo hispánico.

8. Quisiera expresar mi agradecimiento al Dr. L.F. Bernabé por su cuidadosa revisión de mi texto. 


\begin{abstract}
Daniel Defoe published Robinson Crusoe in 1719. Crusoe, in the preliminary adventures he undergoes before reaching his island, has at one stage to flee from captivity in Sallee (Morocco) where he was being held by a "Moor". He makes good his escape in a boat belonging to his master with the help of a boy called Xury who may safely be identified as a Morisco ("Maresco" in the text). But Crusoe, who had promised to make this boy "a great man" ends up by selling him for sixty pieces of eight to a Portuguese captain. The article argues that Defoe's advocacy of improved relations with the Hispanic world make it unlikely that any ironical criticism of Crusoe's behavior was intended.
\end{abstract}

